\title{
Gesetze essen Arztseele auf: Hands off statt on?
}

E in Beitrag in der Ausgabe 2/2013 der O\&R zum —20-jährigen Jubiläum der IGOST zeigt ein Bild des 2012 leider verstorbenen Prof. Jürgen Krämer bei einem Hands-on-Kurs zum Erlernen lumbaler Injektionstechniken. Heutzutage nun stellt sich die Frage, ob Ärzte angesichts des am 26.2.2013 in Kraft getretenen Patientenrechtegesetzes nicht besser die Hände von ihren Patienten lassen sollten. Ein Fall der Norddeutschen Schlichtungsstelle (Niedersächsisches Ärzteblatt 3/2013) gerät zum Menetekel.

Ein 64 Jahre alter Patient mit akuter Lumbago erhielt auf seinen ausdrücklichen Wunsch hin wegen guter Erfahrung mit einer Injektionstherapie und subjektiver Unverträglichkeit von Medikamenten wie NSAR an zwei unterschiedlichen Stellen jeweils intramuskulär Prednisolon und Metamizol appliziert. Die Folgen: 1. Spritzenabszess, 2. Patient verklagt Arzt, 3. Gutachter: Die Fachinformation des verabreichten Prednisolon-Präparats führe als zugelassenes Anwendungsgebiet die Lumbago nicht auf. Somit sei der Glutealabszess die Folge einer nicht indizierten und nicht zugelassenen Injektionsbehandlung. Die Schlichtungsstelle folgt dem Gutachter, da die intramuskuläre Behandlung der akuten Lumbago zudem nicht leitlinienkonform sei.

\section{Was lernen wir daraus?}

1. Leitlinien werden nicht nur von - mangels medizinischen Fachwissens - völlig überforderten Gerichten zu rechtsverbindlichen Richtlinien aufgewertet.

2. Die Beeinflussung der ärztlichen Entscheidung durch Patientenwünsche ist kontraproduktiv, denn: Der Hilfesuchende von heute ist der Kläger von morgen. Das Schicksal in unserer Lebenswelt wird negiert. Alles ist machbar, alles beherrschbar. Entspricht ein Resultat nicht den Erwartungen, ist von einem Fehler und einem Schuldigen auszugehen. Diese naive Vereinfa- chung heterogener, hochkomplexer Wirkungszusammenhänge lähmt alle Handlungen und tötet jegliche Kreativität.

3. Vorsicht, Gutachter! Unabhängig von dem hier geschilderten konkreten Fall scheint es eine Vielzahl völlig untauglicher Gutachter zu geben, die in dieser Betätigung eine lukrative medizinische Weiterbeschäftigung finden - darunter solche, die sich durch angelesenes Wissen befähigt fühlen, auch zu praktischen Fragen interventioneller Techniken gutachterliche, rechtlich relevante Stellungnahmen abzugeben. Andere haben ihre empirischen Fachkenntnisse zu Zeiten von Max Lange (1899-1975) erworben, können aber nicht vom aktuellen Medizingeschäft lassen, weil Verblendung und Hybris eine stete Nachfrage nach ihrer Fachkompetenz suggerieren. Zudem werden Gutachterfehler selten rechtsrelevant.

\section{Werden wir zu Rechtfertigungsmedizinern?} Gemäß $\$ 630$ e BGB ist der Patient über sämtliche, für seine Einwilligung wesentlichen Umstände der geplanten Maßnahme aufzuklären, also über Art, Umfang, Durchführung, Folgen, Risiken, Notwendigkeit, Dringlichkeit, Eignung, Erfolgsaussichten und Alternativen. Diese Aufklärung muss rechtzeitig vor der geplanten Maßnahme, bei elektiven Eingriffen mindestens einen Tag zuvor, stattfinden. Von der unterschriebenen Aufklärung ist dem Patienten eine Abschrift auszuhändigen. Eine Empfangsbestätigung durch den Patienten ist aus beweisrechtlicher Sicht empfehlenswert. Noch Fragen?

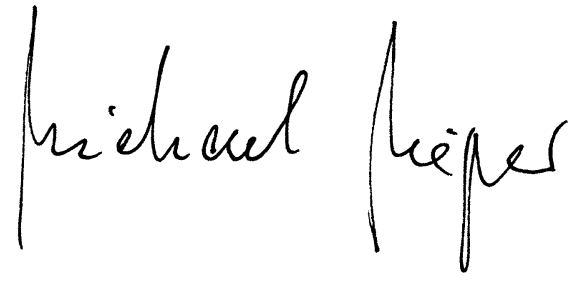

